\title{
Frequency of False Positive Amphetamine Screens due to Bupropion Using the Syva Emit II Immunoassay
}

\author{
Erica R. Casey • Mitchell G. Scott • Schirin Tang • \\ Michael E. Mullins
}

Published online: 30 December 2010

(C) American College of Medical Toxicology 2010

\begin{abstract}
Bupropion is a commonly prescribed, monocyclic antidepressant often used as an aid for smoking cessation. Several case reports have described false positive amphetamine urine drug screens (UDS) associated with bupropion. We sought to determine whether false positive amphetamine UDS due to the use of bupropion would be a frequent occurrence. We conducted an IRB-approved, retrospective chart review of all emergency department patients who underwent UDS between 1 January 2006 and 31 July 2007. All urine samples were screened using Syva EMIT II Plus immunoassay reagents. All positive screens underwent confirmation by gas chromatography (GC). We reviewed the records of patients with positive amphetamine UDS. We documented prescription use of bupropion, other antidepressants, stimulants, antipsychotics, and antihypertensives. We recorded evidence of polysubstance abuse (PSA) as patients who had had a documented diagnosis or laboratory evidence of abuse of at least two substances (drugs or ethanol). Of 10,011 urine drug screens, $362(3.6 \%)$ were positive for amphetamine. GC confirmed amphetamines in $234(65 \%)$, but failed to confirm in 128 (35\%). Among the 234 confirmed, records reflected use of
\end{abstract}

Abstract was presented as a poster at the European Association of Poisons Centres and Clinical Toxicologists XXIII International Congress in Seville, Spain, 7-9 May 2008

E. R. Casey $\cdot$ S. Tang $\cdot$ M. E. Mullins $(\bowtie)$

Division of Emergency Medicine,

Washington University School of Medicine,

Campus Box 8072, 660 S. Euclid Avenue,

Saint Louis, MO 63110, USA

e-mail: mullinsm@wusm.wustl.edu

\section{G. Scott}

Division of Laboratory and Genomic Medicine,

Washington University School of Medicine,

Saint Louis, MO, USA bupropion in three $(1.3 \%)$, other antidepressants in 38 $(16 \%)$, antipsychotics in $17(8 \%)$, and amphetamine in 50 (21\%). Records indicated evidence of PSA in $55(24 \%)$. Among the 128 which failed to confirm, records reflected prescription use of bupropion in 53 (41\%). None whose drug screen failed to confirm had evidence of PSA. Therapeutic use of bupropion appears to be the most frequent cause of false positive urine drug screens for amphetamines in our population.

Keywords Bupropion · Urine drug screen - Immunoassay · Interference $\cdot$ False positive

\section{Introduction}

Bupropion (Budeprion, Wellbutrin, Zyban) is an atypical monocyclic antidepressant commonly prescribed in the US for the treatment of major depressive disorder, seasonal affective disorder, adult ADHD, and smoking cessation. The 2009 Red Book ranks Budeprion XL, Budeprion SR, and Wellbutrin XL 57th, 81st, and 87th, respectively, out of the 200 most common brand named medications dispensed in 2008. Bupropion XL, bupropion SR, and bupropion ER ranked 133rd, 134th, and 171st, respectively, among the 200 most common generic prescription drugs dispensed in the same time period. All forms of bupropion combined totaled 20,745,363 prescriptions in the US in 2008 [1].

The therapeutic properties of bupropion are ascribed to the drug's inhibition of the reuptake of norepinephrine and to a lesser extent, dopamine and serotonin. The aminoketone structure of bupropion is metabolized in the liver by the cytochrome P450 isoenzyme CYP2B6 to the active metabolites $R, R$-hydroxybupropion, $S, S$-hydroxybupropion, threohydrobupropion, and erythrohydrobupropion, which 
are responsible for the pharmacological activity of bupropion [2]. Bupropion, erythrohydrobupropion and threohydrobupropion, have chemical structures similar to amphetamine which may result in cross-reactivity with antibodies used in urine drug screen immunoassays (Fig. 1).

Three case reports demonstrated false positive amphetamine urine drug screens associated with bupropion [2]. There are currently no published studies in the literature which have evaluated the incidence of false positive amphetamine screening associated with therapeutic use of bupropion. The purpose of this study was to determine whether false positive urine drug screens due to the use of bupropion would be a frequent occurrence.

\section{Methods}

We conducted an IRB-approved, retrospective chart review of patients presenting to the emergency department (ED) at Barnes-Jewish Hospital between 1 January 2006 and 31 July 2007. We identified all urine drug screens (UDS) performed by our hospital laboratory during this time. UDS was routinely ordered for all level I trauma patients and for all patients requiring psychiatric evaluation. Other patients underwent UDS at the discretion of the treating physician. Urine samples were screened for amphetamine, barbiturates, benzodiazepines, cocaine, opiates, THC metabolites, or phencyclidine using Syva EMIT II Plus immunoassay (Dade Behring, Inc., San Jose, CA) on the Siemens Dimension analyzer. In our hospital, all positive UDS results routinely undergo confirmatory testing. During the period studied, positive amphetamine screens underwent confirmatory testing by gas chromatography/mass spectrometry using Hewlett Packard 5890 with a SPB 35 column. Gas chromatography (GC) results were "confirmed positive" if GC detected dextroamphetamine, methamphetamine, or 3,4-methylenedioxymethampthetamine. We<smiles>CC(N)Cc1ccccc1</smiles><smiles>CC(NC(C)(C)C)C(=O)c1cccc(Cl)c1</smiles><smiles>C[C@H](NC(C)(C)C)[C@H](O)c1cccc(Cl)c1</smiles><smiles>C[C@H](NC(C)(C)C)[C@H](O)c1cccc(Cl)c1</smiles>

Fig. 1 Structures of amphetamine, bupropion, erythrohydrobupropion, and threohydrobupropion, adapted from Wikipedia (http://en. wikipedia.org/wiki/Amphetamine and http://en.wikipedia.org/wiki/ Bupropion, last accessed 9 Sep 2010) reviewed the entire electronic medical record (EMR) at our institution of the ED patients with positive amphetamine screens, including those with results confirmed and not confirmed by GC. We documented the prescription use of selected drugs including bupropion, other antidepressants, stimulants, antipsychotics, and anti-hypertensives. From the EMR, we recorded evidence of polysubstance abuse as patients who had previously tested positive for or had a documented diagnosis of abuse of any two substances (drugs or ethanol). We also performed a post-hoc Fisher's exact test on the distribution of our results.

\section{Results}

Between 1 January 2006 and 31 July 2007, 10,011 urine drug screens were performed by the outpatient laboratory. Out of 10,011 screens, $362(3.6 \%)$ screens were positive for amphetamine. Of those, $234(65 \%)$ were confirmed positive for amphetamines by GC and 128 (35\%) failed to confirm. Among the 234 confirmed positives, records reflected documented prescription use of bupropion in three patients (1.3\%), other antidepressants in 38 patients $(16 \%)$, antipsychotics in 17 patients $(8 \%)$, and amphetamine in 50 patients $(21 \%)$. Records indicated evidence of polysubstance abuse in 55 patients (24\%) (Fig. 2).

Among the 128 who failed to confirm for amphetamines by GC, records documented prescription use of bupropion in 53 patients $(41 \%)$, other antidepressants in 17 patients $(13 \%)$, antipsychotics in 14 patients $(11 \%)$, and labetalol in nine patients $(7 \%)$. No patient whose drug screen failed to

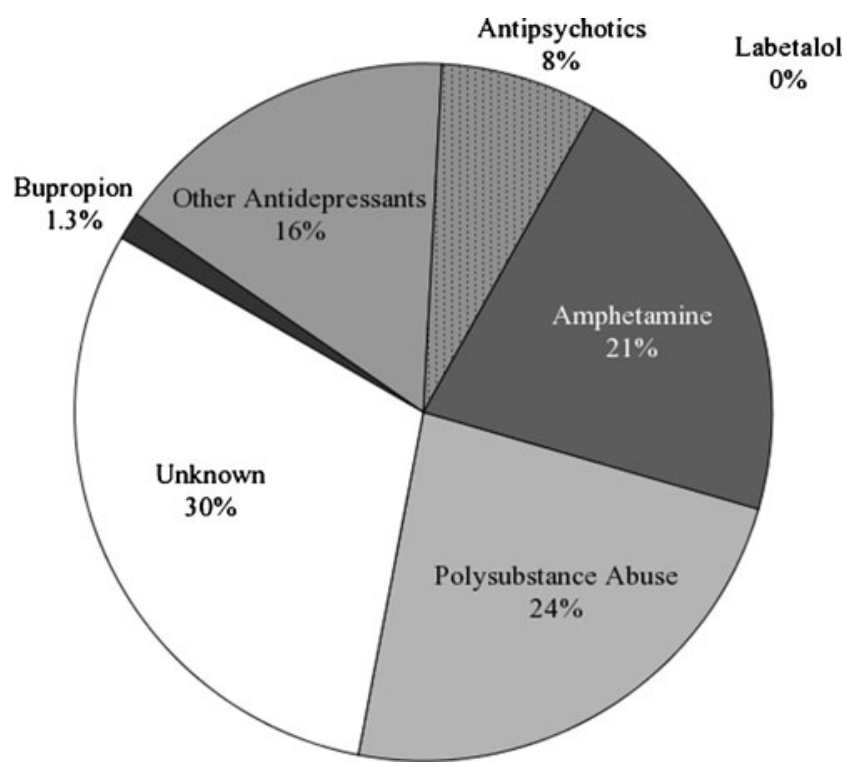

Fig. 2 Distribution of recorded medication and drug use by patients with confirmed positive amphetamine screens 
confirm the presence of amphetamines had evidence of polysubstance abuse (Fig. 3).

\section{Discussion}

From the findings of our retrospective chart review, $41 \%$ of the false positive amphetamine urine drug screens occurred in patients prescribed bupropion. We were struck by the fact that nearly all positive amphetamine screens among patients with identified bupropion use were false positives and that all positive amphetamine screens among patients with identified polysubstance abuse were true positives (Tables 1 and 2). A post-hoc Fisher's exact test found that the distributions of bupropion users or those with evidence of polysubstance abuse were each unlikely to be due to chance alone (bupropion, two-sided $p<0.001$; polysubstance abuse, two-sided $p<0.001$ ). Our results suggest that a large proportion of false positive amphetamine drugs screens by the Syva EMIT II immunoassay are caused by therapeutic administration of bupropion. Furthermore, given over 20 million prescriptions annually for bupropion in the US and the frequent use of urine drug screens in medical, legal, and employment-related settings, the application of our results indicates that significant proportions of patients taking bupropion will elicit a false positive amphetamine urine drug screen.

The limitation of our study is our reliance on medical records, which may be incomplete or inaccurate due to patients' failure to report changes in their prescriptions or

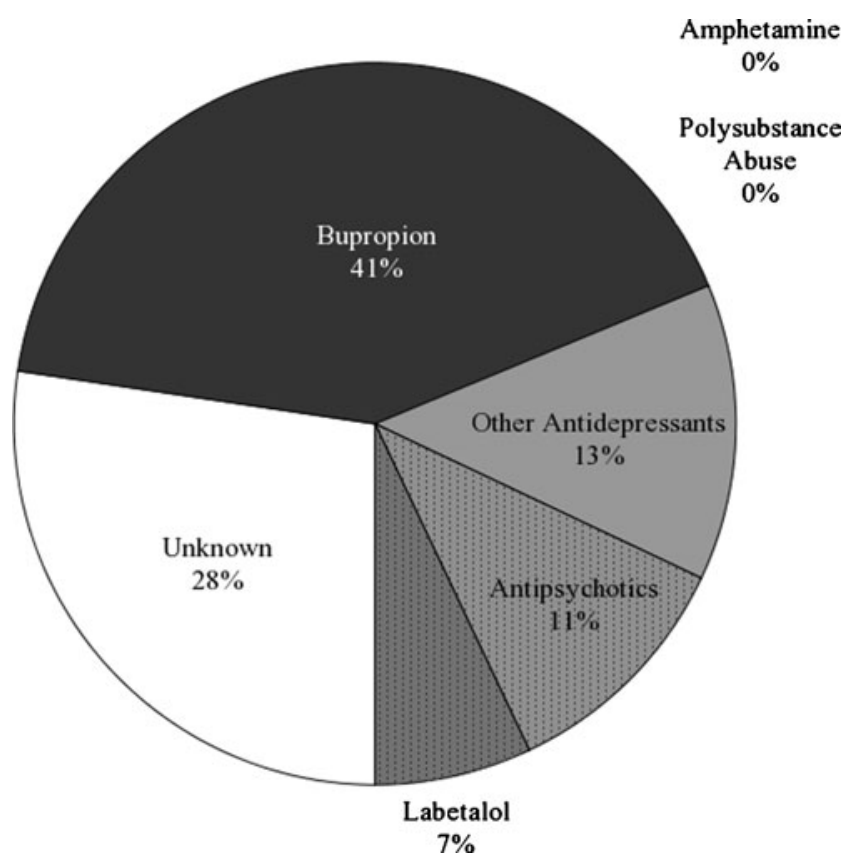

Fig. 3 Distribution of recorded medication and drug use by patients with positive urine drug screens which failed confirmatory testing
Table 1 Frequency of recorded bupropion use by outcome of confirmatory testing

Positive amphetamine screens vs bupropion use

\begin{tabular}{lcrrr}
\hline & & \multicolumn{3}{c}{ Bupropion use } \\
\cline { 3 - 5 } & & \multicolumn{1}{c}{ Yes } & No & Total \\
\hline Confirmed positive & Yes & 3 & 231 & 234 \\
Amphetamine screens & No & 53 & 75 & 128 \\
Total & & 56 & 306 & 362 \\
\hline
\end{tabular}

failure to document use of medication during evaluation of the patient. The population of patients who present to the ED may not always be able to reconcile their medication lists or do not bring their prescriptions for review; therefore our results could underestimate the true percentage of false positive amphetamine screens which are attributable to bupropion.

We also could not confirm compliance with bupropion, which likewise would alter the results of our study. Furthermore, the frequency that our study found may not be representative of the population as a whole since we sampled from an urban level 1 trauma center. Further studies, with voluntary urine drug screening and perhaps corresponding plasma concentrations in patients who are only taking a therapeutic dose of bupropion, could further clarify the degree to which bupropion is responsible for false positive amphetamine screens in our patients.

Finally, we cannot determine whether other drug screen immunoassays would also result in false positive amphetamine results. The EMIT II immunoassay has been previously shown to cross-react with other common psychotropic drugs and may not be a selective enough for amphetamine/methamphetamine [3]. However, the EMIT II immunoassay is a common UDS method and is used by $41 \%$ of the laboratories $(567 / 1,386)$ participating in the July 2010 College of American Pathologists Proficiency Testing Program for urine drug screening [4]. The three previous case reports [5-7] involved different immunoassays, including the Syva EMIT II [5], cloned enzyme donor immunoassay (CEDIA) [6] and EMIT U Amp followed

Table 2 Frequency of recorded polysubstance abuse by outcome of confirmatory testing

Positive amphetamine screens vs evidence of polysubstance abuse

\begin{tabular}{lcrcc}
\hline & & \multicolumn{3}{c}{ Evidence of polysubstance abuse } \\
\cline { 2 - 5 } & & \multicolumn{1}{c}{ Yes } & No & Total \\
\hline Confirmed positive & Yes & 55 & 179 & 234 \\
Amphetamine screens & No & 0 & 128 & 128 \\
Total & & 55 & 307 & 362 \\
\hline
\end{tabular}


by the CEDIA immunoassay [7]. This suggests that crossreactivity by bupropion or its metabolites is not unique to the EMIT II immunoassay.

Bupropion overdose can cause agitation, tremors, hallucinations, tachycardia, or seizures and may clinically resemble amphetamine misuse [8, 9]. This may introduce an ascertainment bias and produce a tendency for bupropion-exposed patients to undergo urine drug screening. Indeed, bupropion overdose itself may be the precipitating event indicating a urine drug screen. However, the cases in this study consistently involved therapeutic use of bupropion and not bupropion overdose.

\section{Conclusion}

Therapeutic use of bupropion appears to be the most frequent cause of false positive urine drug screens for amphetamines in our ED population. Clinicians should be aware of the high false positive rate of the amphetamine screen.

Declaration of interests The authors report no conflict of interests.

\section{References}

1. PDR Network LLC (2009) Red book: Pharmacy's fundamental reference, 2009 edition 113th ed. Thomson PDR, Montvale

2. Hesse L, Venkatakrishnan K (2000) CYP2B6 mediates the in vitro hydroxylation of bupropion: potential drug interactions with other antidepressants. Drug Metab Dispos 28(10):11761183

3. Smith-Kielland A, Olsen KM, Christopherson AS (1995) Falsepositive results with EMIT II amphetamine/methamphetamine assays in users of common psychotropic drugs. Clin Chem 41:951-952

4. UDS-B AACC/CAP Urine Drug Testing (Screening) (2010) College of American Pathologists. IL, Chicago

5. Nixon A, Long W, Puopolo P, Flood J (1995) Bupropion metabolites produce false-positive urine amphetamine results. Clin Chem 41(6):955-956

6. Vidal C, Skripuletz T (2007) Bupropion interference with immunoassays for amphetamines and LSD. Ther Drug Monit 29(3):37333775

7. Weintraub D, Linder MW (2000) Amphetamine positive toxicology screen secondary to bupropion. Depress Anxiety 12(1):53-54. doi:10.1002/1520-6394(2000)12:1<53::AID-DA8>3.0.CO;2-4

8. Jepsen F, Matthews J, Andrews F (2003) Sustained release bupropion overdose: an important cause of prolonged symptoms after an overdose. Emerg Med J 20(6):560-561. doi:10.1136/ emj.20.6.560

9. Shepherd G, Velez L, Keyes D (2004) Intentional bupropion overdoses. J Emerg Med 27(2):147-151. doi:10.1016/j. jemermed.2004.02.017 